\title{
LA SOLIDARITÉ INTERNATIONALE EN FAVEUR DE L'EGLISE CATHOLIQUE DE LA RÉPUBLIQUE DÉMOCRATIQUE DU CONGO
}

\author{
Une forme de mondialisation de la résistance aux régimes autoritaires \\ en Afrique
}

\author{
Par BINAKI BAMANGANA Renia*
}

\section{Résumé}

A la suite la crise politique due à la non tenue des élections à l'échéance constitutionnelle, l'Eglise catholique, par le Comité Laïc de Coordination (CLC), s'est impliquée pour en exiger l'organisation. Ce qui devrait passer par l'application intégrale de l'Accord politique global et inclusif du centre interdiocésain de Kinshasa. L'organisation des manifestations pacifiques tendait à cette fin de faire plier le régime, accusé de confisquer le pouvoir et de l'exercer en dehors des règles constitutionnelles.

Cette réflexion, après avoir montré le fondement de l'intervention de l'Eglise, a indiqué qu'au lieu de faciliter la jouissance de ce droit, les autorités congolaises se sont illustrées par la répression dans le sang de toutes ces manifestations. La disproportion de leur agir a suscité l'indignation et la réprobation internationales des Etats, des organisations internationales et des responsables des églises catholiques des autres Etats dont le Vatican. Les déclarations enregistrées et les actions menées, à cette fin, ont constitué, selon cette étude, une forme mondialisée de lutte contre l'autoritarisme du régime congolais. Cette solidarité internationalisation a impacté positivement le cours des événements et permis au peuple congolais de gagner cette lutte pour la démocratie et l'Etat de droit dans notre pays.

\begin{abstract}
Following the political crisis due to the failure to hold elections on the constitutional deadline, the Catholic Church, through the Lay Coordinating Committee (CLC), became involved in demanding that they be organized. This should be done through the full implementation of the Global and Inclusive Political Agreement of the Inter-diocesan Centre of Kinshasa. To this end, the organization of peaceful demonstrations was aimed at bringing the regime to its knees, accused of confiscating power and exercising it outside the constitutional rules.
\end{abstract}

* Licenciée en Droit et Assistante de recherche au Centre de Recherches et d'Etudes sur l'Etat de Droit en Afrique (CREEDA). 
This reflection, after having shown the basis of the Church's intervention, indicated that instead of facilitating the enjoyment of this right, the Congolese authorities have distinguished themselves by the repression in blood of all these demonstrations. The disproportionate nature of their action has aroused international indignation and reprobation from States, international organizations and Catholic Church leaders in other States, including the Vatican. According to this study, the statements recorded and the actions carried out to this end constituted a globalized form of struggle against the authoritarianism of the Congolese regime. This international solidarity has had a positive impact on the course of events and enabled the Congolese people to win the struggle for democracy and the rule of law in our country.

\section{Introduction}

L'Eglise catholique de la République Démocratique du Congo intervient toujours dans le champ politique. Cela rappelle la question du rapport entre l'ordre spirituel et l'ordre temporel, mieux celle du fondement de cette intervention sur des questions de la sphère civile et non religieuse ${ }^{1}$, intervention mal perçue par les autorités congolaises ${ }^{2}$, même si elle se veut harmonieuse, respectueuse et non une séparation totale, partielle ou hostile d'avec le monde politique, étant donné que l'homme-citoyen dont s'occupe l'Etat est le même dont l'Eglise prend soin au plan spirituel ${ }^{3}$.

La doctrine sociale de l'Eglise en est le fondement, grâce auquel les évêques congolais ont produit un discours en faveur de la démocratie et de l'Etat de droit. Ce qui a permis qu'un évêque dirige la Conférence nationale souveraine en 1992 et que l'Eglise catholique facilite les négociations de l'Accord politique global et inclusif du centre interdiocésain de Kinshasa, dont la mauvaise application est désapprouvée par la CENCO. Le Comité Laïc de Coordination (CLC), mis à profit, en revendique l'application intégrale par l'organisation des manifestations pacifiques, malheureusement réprimées par les autorités ${ }^{4}$.

Cette répression a suscité l'indignation et la solidarité internationale. Cette manifestation de la solidarité exprime la résistance mondialisée contre l'autoritarisme du régime congolais, boostant en même temps la détermination des citoyens à résister, malgré la répression. Ce qui a fait reculer le régime ${ }^{5}$ et changé les points de vue de certains partenaires de la RDC. Elle illustre l'avantage qu'offre la mondialisation. Car, en comprimant le temps et l'espace, la mondialisation permet à ce que les problèmes des uns préoccupent d'autres

1 Faustin Jovith Mapwar Bashuth, Eglise et Société. Le discours sociopolitique des évêques de la Conférence Episcopale Nationale du Congo (CENCO), Faculté catholique de Kinshasa, 2008, p.11.

2 Joseph Kabila, le point de presse du 26 janvier 2018.

3 Faustin Jovith Mapwar Bashuth, Eglise et Société, p.11, 12 et 15, note 1.

4 « Le Comité Laïc de coordination (CLC) appelle à une marche dite de libération du pays », http://w ww.congoactuel.com, consulté le 03 février 2018.

5 Laurel Kankole, " pas d'annonce choc, selon le G7 », http://www.forumdesas.org, consulté le 16 mai 2018. 
qui contribuent ainsi à leur résolution, comme c'est le cas du contexte de l'autoritarisme ambiant dans beaucoup des pays africains, caractérisés par une démocratie de « façade ${ }^{6}$.

Pour le montrer, cette étude expose les revendications légitimes du peuple congolais à travers les marches organisées par le CLC, le comportement disproportionné des autorités politiques congolaises et examine l'incidence de la manifestation de la solidarité internationale considérée comme l'expression mondialisée de la lutte contre cet autoritarisme et un soutien au peuple congolais.

\section{A. Confrontation entre l'Eglise catholique et le régime au pouvoir}

Cette confrontation résulte, malheureusement, de l'insouciance des autorités politiques face aux revendications citoyennes légitimes. Cette situation déplorable ne permet nullement d'envisager une relation de coopération en faveur du bien-être social.

\section{Les revendications fondant l'action de l'Eglise catholique}

Le CLC s'est illustré par de revendications allant de l'amélioration des conditions sociales à l'application intégrale de l'Accord, considéré comme l'unique feuille de route solidement fondée sur la constitution pour des élections crédibles mettant fin à la crise politique actuelle de légitimité 7 .

Pour cette structure ecclésiale, l'application intégrale de l'Accord permettra de libérer l'avenir du pays et en faire une terre de paix, de dignité, d'hospitalité et de progrès pour tous. Elle consiste à exiger au Président de la République la déclaration publique de ne plus être candidat ${ }^{8}$, exigence fondée sur la disposition constitutionnelle selon laquelle le Président de la République est élu (...) pour un mandat de cinq ans renouvelable une seule fois ${ }^{9}$. Ainsi, tout président qui a épuisé le deuxième et dernier mandat ne peut plus en briguer un troisième, comme c'est le cas pour l'actuel Président dont la déclaration devant le congrès a été actée ${ }^{10}$, en dépit de sa volonté de rester au pouvoir à tout prix ${ }^{11}$, inquiétude qu'il n'a pas éclaircie lors de son dernier point de presse $^{12}$.

6 Kä Mana, «Sur le continent africain. En finir avec les démocraties de façade », Congo-Afrique, $\mathrm{n}^{\circ}$ 405, 2006, p. 143.

7 « Le Comité Laïc de coordination (CLC) appelle à une marche dite de libération du pays », note 4.

8 « Le Comité Laïc de coordination (CLC) appelle à une marche dite de libération du pays », note 4.

9 Article 70, al.1 de la Constitution de la République Démocratique du Congo du 18 février 2006 telle que modifiée par la loi n¹1/002 du 20 janvier 2011 portant révision de certains articles de la Constitution de la République Démocratique du Congo du 18 février 2006.

10 Point II.2, III.2.1 de 1'Accord politique global et inclusif du centre interdiocésain de Kinshasa du 31 décembre 2016.

11 Laurel Kankole, « pas d'annonce choc, selon le G7 », note 5.

12 Joseph Kabila, le point de presse du 26 janvier 2018, note 2. 
Mais, cette application concerne surtout les mesures de décrispation qui consistent à l'arrêt des poursuites, à la libération des prisonniers politiques et d'opinion et à la fin de l'exil des réfugiés politiques menacés d'arrestation à leur retour au pays, pour offrir à tous les prétendants les mêmes chances de concourir. Il en est de même du rétablissement du signal des entreprises audiovisuelles fermées ou interdites de fonctionner pour garantir à tous les courants politiques l'égalité d'accès aux médias publics ${ }^{13}$.

Toutes ces mesures sont restées presque lettre morte, puisque certains médias restent toujours fermés et l'accès aux médias publics à tous les courants politiques un veux pieux, aucune mesure prise pour libérer ou cesser de poursuivre les personnalités emblématiques dont certains ne savent pas rentrer au pays ${ }^{14}$.

Si les recommandations faites au ministère de l'intérieur d'instruire les autorités territoriales de plus entraver les activités des organisations politiques ${ }^{15}$ ont quelque peu été satisfaites avec la tenue sans bavure des meetings de l'UDPS et de la plate-forme politique " Ensemble », beaucoup reste encore à faire. C'est le cas du dédoublement dont souffrent encore certains partis, tels que l'UDPS.

Face à l'insouciance des politiques qui ont, du reste, confisqué les ressources nationales et privatisé l'ensemble de services de $1^{\prime}$ Etat $^{16}$, le CLC a aussi exigé l'amélioration des conditions sociales des congolais. Pour toutes ces revendications, les autorités congolaises ont choisi la manière forte consistant à les réprimer, au lieu d'être à l'écoute portant ainsi atteinte à leurs droits fondamentaux.

\section{La réaction disproportionnée des autorités politiques}

Si le droit de manifestation pacifique et d'exprimer librement ces opinions ${ }^{17}$ est garanti, le plus important, c'est d'en assurer la jouissance effective. Ce qui n'a pas été le cas lors des manifestations initiées par le CLC. Car, au lieu de les encadrer, les autorités les ont réprimés de façon disproportionnée.

Elle a d'abord consisté à les interdire ${ }^{18}$, alors que la tentative d'entrave est prohibée, les autorités étant appelée à veiller à leur bon déroulement. La seule intervention requise pour les forces de l'ordre consiste à disperser les manifestants en cas de débordement ou de

13 Point V.2 et 3 de l'Accord politique global et inclusif du centre interdiocésain de Kinshasa, note 10.

14 Kankole, «pas d'annonce choc, selon le G7 », note 5.

15 Point V.4, 5, 6 et 9 de l'Accord politique global et inclusif du centre interdiocésain de Kinshasa, note 10 .

16 «Le Comité Laï de coordination (CLC) appelle à une marche dite de libération du pays », note 4.

17 Articles 23 et 26 de la Constitution de la République Démocratique du Congo du 18 février 2006, note 9.

18 Florence Morice, «RDC : le gouvernement interdit la marche des laïcs catholiques », http://www.r fi.fr, consulté le 04 février 2018 ; «En RDC, la marche catholique anti-Kabila interdite », https://w ww.la-croix.com, consulté le 04 février 2018 ; « Kananga, le maire maintient l'interdiction des manifestations publiques », https://www.radiookapi.net, consulté le 04 février 2018. 
troubles graves ${ }^{19}$, qui n'ont pas été signalés à l'occasion. Aucune disposition ne consacre la répression, telle que vécue. D'ailleurs, le régime juridique des manifestations publiques impose aux organisateurs d'en informer par écrit l'autorité administrative compétente et non solliciter une autorisation, sans violer la constitution ${ }^{20}$. Le comportement outrancier des autorités a violé et porté atteinte au droit à la vie, sacrée pourtant pour être respectée et protégée par l'Etat ${ }^{21}$. Quatorze personnes tuées ont été recensées, dont sept en décembre 2017 et en janvier 2018. Douze l'ont été par balles et deux par gaz lacrymogènes ${ }^{22}$, sans compter le cas Rosy Mukendi, fauché en février 2018.

Le droit à la santé a été atteint par la mise en danger de la vie de nouveau-nés et des malades par l'attaque par gaz lacrymogène de certains hôpitaux et maternités, sans oublier le droit à la liberté de religion entravé par l'inaccessibilité des lieux de cultes, scellés sans décision judiciaire et même vandalisés. Les arrestations illégales ont été opérées et une atteinte au droit à la liberté de mouvement due à l'érection des barrières policières. La liberté d'expression et celle à l'information a été compromise par l'interruption de fournitures de service internet et $\mathrm{SMS}^{23}$.

Cette répression a fait l'objet de dénonciation à l'interne ${ }^{24}$ et d'indignation, sinon de réprobation internationale en soutien aux manifestants.

\section{B. Manifestations de la solidarité internationale en faveur de l'Eglise catholique et son incidence sur la décrispation du climat politique en RDC}

Les images de la répression de ces manifestations, diffusées sur les chaînes de télévision et dans les réseaux sociaux, ont provoqué l'indignation et la réprobation internationales des Etats, des organisations internationales et des responsables des églises catholiques d'autres Etats, à commencer par l'Etat du Vatican. Les différentes déclarations et actions menées, à cet effet, sont une forme mondialisée de lutte contre cet autoritarisme du régime congolais,

19 Articles 7 et 8 du Décret-loi n ${ }^{\circ} 196$ portant réglementation des manifestations et des réunions publiques, JORDC, nº spécial, février 1999.

20 Article 221 de la Constitution de la République Démocratique du Congo du 18 février 2006, note 9.

21 Article 16 de la Constitution de la République Démocratique du Congo du 18 février 2006, note 9.

22 Djaffar al Katanty, «RDC : la commission d'enquete mixte-3121 rend son rapport sur les manifestations du 31 décembre et 21 janvier », http://globalinfos.net, consulté le 02 mai 2018 ; « Ministère des droits humains, rapport synthèse de la Commission d'enquête mixte-3121 », http://www.rf i.fr, consulté le 02 mai 2018.

23 Djaffar al Katanty, « RDC : la commission d'enquête mixte-3121 rend son rapport sur les manifestations du 31 décembre et 21 janvier »; « Ministère des droits humains, rapport synthèse de la Commission d'enquête mixte-3121», note 22 .

24 «Haut-Katanga : 12 ONG dénoncent les « répressions répétitives » des manifestations publiques », https://www.radiookapi.net, consulté le 04 février 2018 ; «L'archevêque de Kinshasa dénonce la barbarie et la médiocrité », https://afrique.lalibre.be, consulté le 03 février 2018. 
solidarité internationalisation qui a impacté le comportement des autorités politiques et les éléments de l'ordre sous leur commandement.

\section{Déclarations et actions de soutien à l'Eglise et aux chrétiens de la RDC}

La première réaction est celle du Pape François, parlant aux diplomates accrédités au Vatican pour que la Communauté internationale prenne en compte les souffrances des peuples des pays africains notamment des Congolais. Aussi, déclare-t-il :

Que la communauté internationale n'oublie pas non plus les souffrances de nombreuses parties du continent africain, spécialement au Sud-Soudan, en République Démocratique du Congo, en Somalie, au Nigéria et en République Centrafricaine, où le droit à la vie est menacé par l'exploitation abusive des ressources, par le terrorisme, par la prolifération de groupes armés et par des conflits persistants. Il ne suffit pas de s'indigner face à tant de violence. Il faut plutôt que chacun, dans son domaine propre, ouvre activement pour éradiquer les causes de la misère et pour construire des ponts de fraternité, condition fondamentale d'un développement humain authentique $^{25}$.

Cette déclaration pontificale manifeste la solidarité de l'Eglise catholique, au plus haut sommet, aux chrétiens catholiques de la RDC. Elle a été suivie d'autres messages de soutien des conférences épiscopales de Belgique et de France adressés au Cardinal Laurent Monsengwo, victime de propos indécents de la part des autorités politiques et de leurs partisans.

Dans son communiqué du 11 janvier 2018, la Conférence Épiscopale de Belgique soutient les évêques congolais dans leur inlassable quête d'une solution pacifique et consensuelle à la crise politique et électorale. Elle rappelle aussi au monde ces efforts de la CEN$\mathrm{CO}$ ayant abouti à l'accord de la Saint Sylvestre, non respecté dans son application intégrale tel que le réclament les chrétiens catholiques réprimés. Ainsi, peut-on lire :

La situation politique en République Démocratique du Congo est extrêmement tendue. Les évêques congolais ont à plusieurs reprises appelé au respect des accords de la Saint-Sylvestre ces derniers mois, accords obtenus grâce à leur médiation, prévoyants des élections présidentielles en fin d'année 2017. Hélas, en vain. L'Eglise catholique du Congo est de plus en plus poursuivie par une partie des pouvoirs publics. Selon les Nations Unies, le 31 décembre, cinq participants à une manifestation en faveur de la démocratie ont été tués par balles. Des célébrations eucharistiques ont été perturbées par des jets de gaz lacrymogène, des dizaines de paroisses ont été attaquées et pillées, de nombreux fidèles ont été spoliés et intimidés. Le Cardinal

25 Patrick Maki, « Devant des diplomates en poste au Vatican, le Pape attire 1'attention sur la situation en RDC », http://actualité.cd, consulté le 9 janvier 2018. 
Laurent Monsengwo Pasinya, archevêque de Kinshasa, parle dans un Communiqué de situations barbares et condamne sévèrement ces événements ${ }^{26}$.

Les évêques belges en ont appelé au respect des droits et libertés fondamentales de chaque être humain au Congo. Ils ont, en outre, invité l'Union Européenne dont le siège se trouve à Bruxelles et la Communauté internationale à s'engager 'sans tarder avec tous les moyens qui sont en leur possession' pour contribuer à la protection des droits et des libertés des Congolais.

Les évêques de France ont, quant à eux, soutenu le Cardinal Monsengwo, figure emblématique de cette contestation. Dans la lettre lui adressée en date du 10 janvier 2018, le Président de la Conférence des évêques de France (CEF) s'exprime en ces termes :

Au moment où des manifestations viennent d'être réprimées par un usage inconsidéré de la force publique, où des paroisses ont été encerclées et des célébrations eucharistiques perturbées en violation de tout respect de la liberté religieuse, avec des victimes nombreuses, vous avez courageusement défendu le droit des catholiques de votre diocèse à manifester pacifiquement et à exprimer leur opinion. Je voudrais redire la solidarité de la Conférence des évêques de France, en ces heures difficiles de l'histoire de votre pays. Nos prières vous accompagnent sur le chemin du rétablissement de l'État de droit au Congo ${ }^{27}$.

Toutes ces déclarations ont été suivies de quelques actions manifestant la solidarité internationale aux chrétiens catholiques congolais. Ce qui a contribué à les encourager à poursuivre leur combat héroïque pour l'établissement de l'Etat de droit dans leur pays. La première action est l'adresse du Nonce apostolique aux évêques et archevêques de la RDC. Dans sa lettre du 5 janvier 2017, concernant les futures manifestations des chrétiens catholiques après celles du 31 décembre 2017, le Nonce soutenait la poursuite de telles actions:

Tenant compte de la popularité de la marche du 31 décembre 2017, il est fort probable que d'autres initiatives seront organisées dans les prochains mois. Il faut se préparer à répondre aux éventuelles adhésions dans votre circonscription ecclésiastique ${ }^{28}$. Cette lettre les poussait à l'action en commençant par leur rassurer du statut canonique du CLC, qui est bel et bien une structure légalement établie au sein de l'Eglise catholique de Kinshasa, conformément au droit canonique. Cette assurance permettait de lever le doute qui serait à base de l'inaction de certains évêques.

Cette lettre les poussait à l'action en commençant par leur rassurer du statut canonique du CLC, qui est bel et bien une structure légalement établie au sein de l'Eglise catholique

26 «RDC: Les évêques belges apportent leur soutien à la CENCO », https://actualite.cd/2018/01/11/r dc-eveques-belges, consulté le 12 janvier 2018.

27 « Lettre de Monseigneur Georges Pontier au Cardinal Laurent Monsengwo », https://eglise.catholi que.fr/actualites/451343-451343/, consulté le 05 juillet 2018.

28 «RDC: vers de probables nouvelles manifestations des chrétiens, signale la Nonce apostolique », https://actualite.cd/2018/01/06/rdc-vers-de-probables-nouvelles, consulté le 6 janvier 2018. 
de Kinshasa, conformément au droit canonique. Cette assurance permettait de lever le doute qui serait à base de l'inaction de certains évêques.

Je tiens à vous informer que le CLC a demandé auprès de Cardinal Archevêque de Kinshasa la nécessaire recognition de ses statuts pour être constitué canoniquement comme Association privée. Cette recognition lui a été donnée de manière provisoire et par conséquent, le CLC a une personnalité juridique et peut organiser ses initiatives dans tout le territoire de l'Archidiocèse ${ }^{29}$.

Cette lettre a été déterminante dans le processus d'adhésion des chrétiens catholiques de différents diocèses de la RDC aux manifestations organisées par le CLC. Cette action n'a pas plu aux autorités congolaises qui s'apprêtaient à le déclarer persona non grata, sans y parvenir le Saint Siège l'ayant rappelé entretemps.

La deuxième action est l'appel au jeûne et à la prière lancé le 4 février 2018 par le Pape aux chrétiens et non chrétiens en faveur de la paix en RDC. Cette invitation du Souverain Pontife s'adressait aux chrétiens catholiques et aux autres confessions religieuses, pour une Journée de jeûne et de prière le vendredi 23 février 2018 en faveur de la paix en République Démocratique du Congo et au Soudan du Sud. Une Journée consacrée aux populations des deux pays en pleine crise politique avec des conséquences directes sur les populations. Cet appel a été motivé par la « ...poursuite tragique de la situation de conflit... » dans les Etats visés. Ce vendredi 23 février, tous les fidèles catholiques du monde avaient partagé les peines endurées par les populations congolaises ${ }^{30}$.

L'enseignement du Pape François sur la politique constitue la troisième action. Deux leçons peuvent se dégager pour encourager les catholiques congolais dans leur lutte pour le changement démocratique. Pour lui, un bon catholique doit donner le meilleur de lui-même. Il doit donner des idées, des conseils et doit prier pour que le gouvernant puisse gouverner correctement. La prière à elle seule ne suffit pas. Un catholique doit s'impliquer dans la politique pour contribuer à l'établissement d'un régime qui respecte la dignité humaine. Voici en cela sa déclaration:

Qui gouverne,..., doit aimer son peuple, car un gouvernant qui n'aime pas, ne peut pas gouverner : au maximum il pourra discipliner, mettre un peu d'ordre, mais pas gouverner ». Mais le bon catholique, lui " ne doit pas se désintéresser de la politique », il doit au contraire, "pour le bien de tous (...) faire son possible pour que le gouvernant gouverne bien, en participant de son mieux à la vie politique ${ }^{31}$.

29 «RDC: vers de probables nouvelles manifestations des chrétiens, signale la Nonce apostolique », note 28

30 «RDC : La CENCO a publié le programme de la Journée de jeûne et de prière appelée par la Pape François », https://actualite.cd/2018/02/22/rdc-cenco, consulté le 22 février 2018.

31 Cousturié, «Pape François : un bon catholique doit se mêler de politique », https://fr.aleteia.org/20 13/09/16/pape-francois, consulté le 05 juillet 2018. 
Toutes ces déclarations et actions ont eu de l'incidence sur l'action des autorités politiques congolaises décrispant tant soit peu le climat politique délétère, sur certains partenaires de la RDC et même encouragé cette résistance des chrétiens congolais.

\section{Incidence de l'action internationale sur la décrispation du climat politique en RDC}

L'impact de cette solidarité internationale sur les autorités congolaises est remarquable à travers l'action de trois ministres que sont les ministres de l'intérieur, des droits humains et de la justice. Du côté du ministère de l'intérieur, le nouveau Vice-premier ministre et ministre de l'intérieur a lors de la cérémonie de sa présentation aux officiers de la police nationale congolaise déclaré que:

Les civils ne sont donc pas de combattants ennemis même lorsqu'ils ont violé la loi. Même en cas d'émeute ou de tentative d'insurrection, les civils sont des compatriotes qui, momentanément, ont perdu le sens de la citoyenneté. C'est donc des égarés qu'il faut ramener à la raison. Il faudra ainsi les contenir, les canaliser, les redresser, les aiguiller, les conduire, les conseiller, etc. pour les soumettre au respect de la loi en utilisant le minimum de violence "...la police doit être en pleine complicité avec le peuple de qui elle a reçu mission. C'est donc une erreur inqualifiable de se mettre à dos le peuple de même qu'il est dangereux pour la concorde nationale que les citoyens cessent d'en appeler à sa police ${ }^{32}$.

Cette déclaration n'avait pas été prise au sérieux, quand on sait que quelque temps auparavant, ce ministre alors ancien secrétaire général du parti présidentiel avait fait former des jeunes (bérets rouges) qu'il avait envoyé occuper l'enceinte de la Cathédrale Notre Dame du Congo à la veille d'une marche pacifique annoncée par le CLC. On pensait qu'il s'agissait d'une annonce pour la consommation de masse. Mais, sa déclaration s'est matérialisée lors des rassemblements organisés par les partis politiques de l'opposition, en l'occurrence l'Union pour la Démocratie et le Progrès Social (UDPS) et le regroupement politique 'Ensemble', soutenant Moise Katumbi à l'élection présidentielle. Aucun incident n'a été déploré qui impliquerait la police et les forces de l'ordre. On n'y a, par ailleurs, enregistré aucune personne tuée, même pas blessée.

Ces deux exemples illustrent mieux la nécessité de décrisper le climat politique pour espérer avoir les bonnes élections qui soient aussi apaisées. Cette attitude des forces de l'ordre et de sécurité est une des retombées de la solidarité internationale en faveur des chrétiens catholiques.

Le deuxième ministre est celui des Droits humains. A son actif, la mise sur pieds, depuis le 5 février 2018, d'une commission d'enquête mixte-3121, composée des délégués de son ministère, de ceux du ministère de la Justice, de la Commission Nationale des Droits de

32 Tshibuyi, «RDC: Mova appelle la police à ne pas considérer des civils comme " ennemis 》", https://actualite.cd/2018/03/23/rdc, consulté le 23 mars 2018. 
l'homme (CNDH), de la société civile, du Bureau Conjoint des Nations Unies aux Droits de l'homme (BCNUDH) et du Bureau de liaison de l'Union Africaine (UA) pour enquêter sur les allégations d'atteinte aux droits humains lors des manifestations organisées par le CLC.

A l'issue des travaux de collecte, de vérification desdites allégations, le rapport remis au ministre de la justice ${ }^{33}$ accuse les forces de l'ordre d'avoir recouru à l'usage disproportionné de la force. Et, pour cette commission, ce rapport devrait servir de base aux enquêtes judiciaires à l'encontre des présumés auteurs et complices, contribuant ainsi à lutter contre l'impunité en $\mathrm{RDC}^{34}$.

Ce à quoi le ministre de la justice s'engage. Il promet de saisir le PGR en lui faisant injonction contre les auteurs présumés de la répression des manifestations. Pour ce membre $\mathrm{du}$ gouvernement congolais, ce sont les efforts gouvernementaux pour sanctionner les responsables de telles atteintes. L'adoption du principe de la tolérance zéro vis-à-vis des militaires montre à suffisance, selon le ministre de la justice, que l'exécutif congolais fait beaucoup de choses, contrairement à toutes les propagandes faites contre le pays et par rapport à d'autres non indexés par les rapports de la communauté internationale ${ }^{35}$.

Telle est l'incidence de la solidarité internationale en soutien aux manifestants que nous avons mis en exergue concernant le gouvernement congolais. Quant au revirement des points de vue diplomatiques chez certains partenaires de la RDC, l'exemple de la France et de l'Angola est assez illustratif. En effet, depuis son arrivée au pouvoir, le Président français, Emmanuel Macron, s'était montré moins préoccupé par la crise congolaise. Sa prise de position à la conférence de presse conjointe du 28 mai 2018 avec le Président angolais pourrait être qualifiée d'un revirement spectaculaire salué par l'opinion publique, bien qu'ayant suscité des réactions diverses au sein du Gouvernement congolais. Il a notamment déclaré:

... la France viendra en soutien des initiatives venant des pays de la sous-région notamment celles de faire appliquer les accords qui, seules, permettront une clarification de la situation politique, sans aucune complaisance, dans le calme et avec clarté, élections auxquelles le président Kabila ne pourra pas participer ...les réunions qui auront lieu dans les prochaines semaines permettront d'aboutir à un résultat clair $^{36}$.

33 Nlemvo, «RDC : Thambwe Mwamba promet de saisir le PGR contre les auteurs de la répression des manifestations ", https://actualite.cd/2018/06/28/rdc-thambwe-mwamba-promet-de-saisir-le-pg r-contre-les-auteurs-de-la-repression-des-manifestations/, consulté le 28 juin 2018.

34 Djaffar al Katanty, «RDC : la commission d'enquête mixte-3121 rend son rapport sur les manifestations du 31 décembre et 21 janvier », note 22 .

35 Nlemvo, «RDC : «tolérance zéro pour les forces de l'ordre responsables des violations des droits de l'homme ", https://actualite.cd/2018/06/28/rdc-thambwe-mwamba-promet-de-saisir-le-pgr-cont re-les-auteurs-de-la-repression-des-manifestations/, consulté le 28 juin 2018.

36 « La RDC de nouveau au menu des entretiens à Paris entre les présidents français et angolais ", http://www.mobile.topcongo.fm, consulté le 28 mai 2018. 
Ce qui fâche, dans cette déclaration accueillie comme un coup d'éclat par le Gouvernement congolais, ce se sont les bouts de phrases comme " initiatives sous régionales » méconnues du gouvernement congolais, « élections auxquelles le Président Kabila ne pourra pas participer $»$.

L'Angola a également changé d'attitude à l'égard du régime de Joseph Kabila qu'il avait soutenu depuis plus d'une décennie. Le Président angolais est devenu l'acteur majeur du revirement diplomatique régional et ne se cache plus pour assumer ce statut. Dans une interview accordée à la Radio France Internationale (RFI), il déclarait que:

$V u$ qu'il y a un accord, les acteurs intérieurs mais aussi les partenaires internationaux, les pays voisins ont tout intérêt à ce qu'il (accord) soit respecté. La communauté internationale, pas seulement l'Angola, a attiré l'attention du président Kabila sur cette nécessité. Pour le moment, le président Kabila affirme que c'est ce qu'il a fait, que le recensement électoral est pratiquement conclu, que des ressources financières ont été allouées aux élections. Donc il faut lui accorder le bénéfice du doute. Nous n'avons pas de raison de penser que l'accord ne sera pas respecté. Nous continuerons de suivre la situation jusqu'en décembre ${ }^{37}$.

Pour tout dire, cette solidarité internationale est une marque très concrète du soutien que les manifestants ont reçu pour finalement se sentir revigorer dans leur effort pour mettre un terme à cette sorte de dictature sournoise qui ne dit pas encore clairement son nom. Ce soutien a galvanisé les organisateurs à poursuivre la pression sur le pouvoir afin d'obtenir notamment le départ de l'actuel Président de la République. Pour preuve et face aux tentatives on ne peut plus claires des partisans de l'actuel chef de l'Etat désireux de le voir briguer un autre mandat, le CLC a projeté des actions d'envergure après la période du dépôt de candidature à la députation nationale et à la présidentielle qui court du 25 juillet au 8 août 2018 .

Entretemps, une banderole avait été hissée à l'enceinte du centre interdiocésain, faisant état du rejet d'une probable candidature du chef de l'Etat en exercice. Aussi, le CLC fait circuler de petits dépliants qui rappellent la prohibition par la Constitution d'un troisième mandat pour le Président sortant. En passant, les universitaires l'ont rappelé récemment.

\section{Conclusion}

L'implication de l'Eglise catholique de la RDC dans la sphère politique vise avant tout l'établissement d'un Etat de droit démocratique. Pour une telle lutte, l'Eglise n'est pas prête à se laisser faire et à se taire, n'en déplaisent aux autorités politiques congolaises caractérisées par l'incurie et l'insouciance quant au besoin de résoudre la situation misérable dans

37 Nlemvo, « João Lourenço: Les pays voisins de la RDC ont tout intérêt à ce que l'accord soit respecté », http://www.rfi.fr, consulté le 31 mai 2018. 
laquelle le peuple vit, contraint à recourir pour survivre à des expédients et aux solutions incompatibles à la conscience ${ }^{38}$.

Cette ténacité de l'Eglise est en partie réconfortée par le soutien international reçu qui a impacté positivement le gouvernement congolais comme démontré, bien qu'il reste encore beaucoup à faire pour une application intégrale dudit Accord. Cette solidarité internationale, expression de la lutte mondialisée contre l'autoritarisme ambiant en RDC et en faveur de la démocratie et de l'Etat de droit, a été un électrochoc pour le changement d'attitude observée chez certains partenaires de la RDC qui ont finalement compris à quel niveau se situe le problème.

Ils n'ont pas seulement condamné la répression sanglante déchainée par les pouvoirs publics sensé promouvoir et protéger les droits fondamentaux de ses citoyens, ils ont surtout posé des actes en soutien aux revendications légitimes exprimées lors de ces manifestations, appelant expressément à l'organisation des élections en décembre 2018, sans la participation du Président actuellement au pouvoir arrivé à la fin de son deuxième et dernier mandat constitutionnel.

A la suite de cette solidarité internationale, considérée comme une forme mondialisée de lutte contre l'autoritarisme du régime congolais, le peuple congolais en est sorti vainqueur au regard de l'aboutissement du processus électoral. Le fait pour le Président sortant de ne s'être pas représenté à la suite d'une modification redoutée de la Constitution est une victoire pour l'émergence de la démocratie congolaise et de l'Etat de droit.

\section{Bibliographie}

I. Textes juridiques

1. Constitution de la République Démocratique du Congo de 2006 telle que modifiée par la loi n $11 / 002$ du 20 janvier 2011 portant révision de certains articles de la Constitution de la République Démocratique du Congo du 18 février 2006.

2. Accord politique global et inclusif du centre interdiocésain de Kinshasa du 31 décembre 2016.

3. Décret-loi $\mathrm{n}^{\circ} 196$ portant réglementation des manifestations et des réunions publiques, JORDC, nº spécial, février 1999.

\section{Ouvrage}

1. Faustin Jovith Mapwar Bashuth, Eglise et Société. Le discours sociopolitique des évêques de la Conférence Episcopale Nationale du Congo (CENCO), Faculté catholique de Kinshasa, 2008.

38 Mapwar Bashuth, Eglise et Société. Le discours sociopolitique des évêques de la Conférence Episcopale Nationale du Congo (CENCO), p.11, note 1. 
III. Article

1. Kä Mana, « Sur le continent africain. En finir avec les démocraties de façade », CongoAfrique, $n^{\circ}$ 405, 2006.

IV. Sites internes consultés

1. «Le Comité Laïc de coordination (CLC) appelle à une marche dite de libération du pays », http://www.congoactuel.com, consulté 03 février 2018.

2. Laurel Kankole, «pas d'annonce choc, selon le G7 », http://www.forumdesas.org, consulté 16 mai 2018.

3. Florence Morice, " RDC : le gouvernement interdit la marche des laïcs catholiques ", http://www.rfi.fr, consulté le 04 février 2018.

4. «En RDC, la marche catholique anti-Kabila interdite », https://www.la-croix.com, consulté le 04 février 2018.

5. «Kananga, le maire maintient l'interdiction des manifestations publiques », https://w ww.radiookapi.net, consulté le 04 février 2018.

6. Djaffar al Katanty, « RDC : la commission d'enquête mixte-3121 rend son rapport sur les manifestations du 31 décembre et 21 janvier », http://globalinfos.net, consulté le 02 mai 2018.

7. "Ministère des droits humains, rapport synthèse de la Commission d'enquête mixte-3121», http://www.rfi.fr, consulté le 02 mai 2018.

8. «Haut-Katanga : $12 \mathrm{ONG}$ dénoncent les « répressions répétitives » des manifestations publiques », https://www.radiookapi.net, consulté le 04 février 2018.

9. «L'archevêque de Kinshasa dénonce la barbarie et la médiocrité », https://afrique.lali bre.be, consulté le 03 février 2018.

10. Patrick Maki, « Devant des diplomates en poste au Vatican, le Pape attire l'attention sur la situation en RDC », http://actualité.cd, consulté le 09 janvier 2018.

11. «RDC: Les évêques belges apportent leur soutien à la CENCO », https://actualite.cd/ 2018/01/11/rdc-eveques-belges, consulté le 12 janvier 2018.

12. « Lettre de Monseigneur Georges Pontier au Cardinal Laurent Monsengwo », https://e glise.catholique.fr/actualites/451343-451343/, consulté le 05 juillet 2018.

13. «RDC: vers de probables nouvelles manifestations des chrétiens, signale la Nonce apostolique », https://actualite.cd/2018/01/06/rdc-vers-de-probables-nouvelles, consulté le 06 janvier 2018.

14. « RDC : La CENCO a publié le programme de la Journée de jeûne et de prière appelée par la Pape François », https://actualite.cd/2018/02/22/rdc-cenco, consulté le 22 février 2018.

15. Cousturié, « Pape François : un bon catholique doit se mêler de politique », https://fr.a leteia.org/2013/09/16/pape-francois, consulté le 05 juillet 2018. 
16. Tshibuyi, «RDC: Mova appelle la police à ne pas considérer des civils comme « ennemis » », https://actualite.cd/2018/03/23/rdc, consulté le 23 mars 2018.

17. Nlemvo, « RDC : Thambwe Mwamba promet de saisir le PGR contre les auteurs de la répression des manifestations », https://actualite.cd/2018/06/28/rdc-thambwe-mwamb a-promet-de-saisir-le-pgr-contre-les-auteurs-de-la-repression-des-manifestations/, consulté le 28 juin 2018.

18. Nlemvo, « RDC : « tolérance zéro pour les forces de l'ordre responsables des violations des droits de l'homme », https://actualite.cd/2018/06/28/rdc-thambwe-mwambapromet-de-saisir-le-pgr-contre-les-auteurs-de-la-repression-des-manifestations/, consulté le 28 juin 2018.

19. « La RDC de nouveau au menu des entretiens à Paris entre les présidents français et angolais », http://www.mobile.topcongo.fm, consulté le 28 mai 2018.

20. Nlemvo, « João Lourenço: Les pays voisins de la RDC ont tout intérêt à ce que l'accord soit respecté », http://www.rfi.fr, consulté le 31 mai 2018.

V. Point de presse

1. Joseph Kabila, le point de presse du 26 janvier 2018. 


\section{Impressum}

\section{KAS African Law Study Library - Librairie Africaine d'Etudes Juridiques}

Herausgeber: Konrad-Adenauer-Stiftung e.V., Klingelhöferstraße 23, 10785 Berlin

Schriftleitung: RA Prof. Dr. Hartmut Hamann, Falkertstraße 82, D-70193 Stuttgart Telefon: +49 (o) 711 120950-30 | Fax : +49 (o) 711 120950-50

Mail:hamann@hamann-legal.de

Erscheinungsweise: 4 Ausgaben pro Jahr

Druck und Verlag: Nomos Verlagsgesellschaft mbH \& Co. KG - Waldseestr. 3-5 D-76530 Baden-Baden. Telefon +49-7221-2104-0 / Fax 49-7221-2104-27 E-Mail:nomos@nomos.de

Anzeigen: sales friendly Verlagsdienstleistungen - Inh. Frau Bettina Roos - Pfaffenweg 15 - D-53227 Bonn Telefon +49-228-97898-o / Fax +49-228-97898-20 E-Mail:roos@sales-friendly.de

Urheber- und Verlagsrechte: Die Zeitschrift und alle in ihr enthaltenen einzelnen Beiträge sind urheberrechtlich geschützt. Jede Verwertung außerhalb der engen Grenzen des Urheberrechtsgesetzes ist ohne Zustimmung des Verlages unzulässig. Das gilt insbesondere für Vervielfältigungen, Übersetzungen, Mikroverfilmungen und die Einspeicherung und Verarbeitung in elektronischen Systemen. Der Nomos Verlag beachtet die Regeln des Börsenvereins des Deutschen Buchhandels e.V. zur Verwendung von Buchrezensionen.

ISSN $2363-6262$

\section{Nomos}

